\title{
ON THE STABILITY OF SOLUTIONS OF LINEAR BOUNDARY VALUE PROBLEMS FOR A SYSTEM OF ORDINARY DIFFERENTIAL EQUATIONS
}

\author{
M. ASHORDIA
}

\begin{abstract}
Linear boundary value problems for a system of ordinary differential equations are considered. The stability of the solution with respect to small perturbations of coefficients and boundary values is investigated.
\end{abstract}

Let $\mathcal{P}_{0}:[a, b] \rightarrow \mathbb{R}^{n \times n}$ and $q_{0}:[a . b] \rightarrow \mathbb{R}^{n}$ be integrable matrix- and vector-functions, respectively, $c_{0} \in \mathbb{R}^{n}$, and let $l_{0}: C\left([a, b] ; \mathbb{R}^{n}\right) \rightarrow \mathbb{R}^{n}$ be a linear continuous operator such that the boundary value problem

$$
\begin{aligned}
& \frac{d x}{d t}=\mathcal{P}_{0}(t) x+q_{0}(t), \\
& l_{0}(x)=c_{0}
\end{aligned}
$$

has the unique solution $x_{0}$. Consider the sequences of integrable matrixand vector-functions, $\mathcal{P}_{k}:[a, b] \rightarrow \mathbb{R}^{n \times n}(k=1,2, \ldots)$, and $q_{k}:[a, b] \rightarrow \mathbb{R}^{n}$ $(k=1,2, \ldots)$, respectively, the sequence of constant vectors $c_{k} \in \mathbb{R}^{n}(k=$ $1,2, \ldots)$ and the sequence of linear continuous operators $l_{k}: C\left([a, b] ; \mathbb{R}^{n}\right) \rightarrow$ $\mathbb{R}^{n}(k=1,2, \ldots)$. In $[1,2]$, sufficient conditions are given for the problem

$$
\begin{aligned}
& \frac{d x}{d t}=\mathcal{P}_{k}(t) x+q_{k}(t), \\
& l_{k}(x)=c_{k}
\end{aligned}
$$

to have a unique solution $x_{k}$ for any sufficiently large $k$ and

$$
\lim _{k \rightarrow+\infty} x_{k}(t)=x_{0}(t) \quad \text { uniformly on }[a, b] .
$$

In the present paper, necessary and sufficient conditions are established for the sequence of boundary value problems of the form $(3),(4)$ to have the above-mentioned property.

1991 Mathematics Subject Classification. 34B05. 
Throughout the paper the following notations and definitions will be used:

$\mathbb{R}=]-\infty,+\infty[$

$\mathbb{R}^{n}$ is a space of real column $n$-vectors $x=\left(x_{i}\right)_{i=1}^{n}$ with the norm

$$
\|x\|=\sum_{i=1}^{n}\left|x_{i}\right|
$$

$\mathbb{R}^{n \times n}$ is a space of real $n \times n$ matrices $X=\left(x_{i j}\right)_{i, j=1}^{n}$ with the norm

$$
\|X\|=\sum_{i, j=1}^{n}\left|x_{i j}\right|
$$

if $X=\left(x_{i j}\right)_{i, j=1}^{n} \in \mathbb{R}^{n \times n}$, then $\operatorname{diag} X$ is a diagonal matrix with diagonal components $x_{11}, \ldots, x_{n n} ; X^{-1}$ is an inverse matrix to $X ; E$ is an identity $n \times n$ matrix;

$C\left([a, b] ; \mathbb{R}^{n}\right)$ is a space of continuous vector-functions $x:[a, b] \rightarrow \mathbb{R}^{n}$ with the norm

$$
\|x\|_{c}=\max \{\|x(t)\|: a \leq t \leq b\}
$$

$\widetilde{C}\left([a, b] ; \mathbb{R}^{n}\right)$ and $\widetilde{C}\left([a, b] ; \mathbb{R}^{n \times n}\right)$ are the sets of absolutely continuous vector- and matrix- functions, respectively;

$L\left([a, b] ; \mathbb{R}^{n}\right)$ and $L\left([a, b] ; \mathbb{R}^{n \times n}\right)$ are the sets of vector- and matrix-functions $x:[a, b] \rightarrow \mathbb{R}^{n}$ and $X:[a, b] \rightarrow \mathbb{R}^{n \times n}$, respectively, whose components are Lebesgue-integrable;

$\|l\|$ is the norm of the linear continuous operator $l: C\left([a, b] ; \mathbb{R}^{n}\right) \rightarrow \mathbb{R}^{n}$.

The vector-function $x:[a, b] \rightarrow \mathbb{R}^{n}$ is said to be a solution of the problem (1),(2) if it belongs to $\widetilde{C}\left([a, b] ; \mathbb{R}^{n}\right)$ and satisfies the condition (2) and the system (1) a.e. on $[a, b]$.

Definition 1. We shall say that the sequence $\left(\mathcal{P}_{k}, q_{k}, l_{k}\right)(k=1,2, \ldots)$ belongs to $S\left(\mathcal{P}_{0}, q_{0}, l_{0}\right)$ if for every $c_{0} \in \mathbb{R}^{n}$ and $c_{k} \in \mathbb{R}^{n}(k=1,2, \ldots)$ satisfying the condition

$$
\lim _{k \rightarrow+\infty} c_{k}=c_{0}
$$

the problem (3),(4) has the unique solution $x_{k}$ for any sufficiently large $k$ and (5) holds.

Along with (1),(2) and (3),(4) we shall consider the corresponding homogeneous problems

$$
\begin{aligned}
& \frac{d x}{d t}=\mathcal{P}_{0}(t) x, \\
& l_{0}(x)=0
\end{aligned}
$$


and

$$
\begin{aligned}
& \frac{d x}{d t}=\mathcal{P}_{k}(t) x, \\
& l_{k}(x)=0 .
\end{aligned}
$$

Theorem 1. Let

$$
\lim _{k \rightarrow+\infty} l_{k}(y)=l_{0}(y) \quad \text { for } y \in \widetilde{C}\left([a, b] ; \mathbb{R}^{n}\right)
$$

and

$$
\lim _{k \rightarrow+\infty} \sup \left\|l_{k}\right\|<+\infty
$$

Then

$$
\left(\left(\mathcal{P}_{k}, q_{k}, l_{k}\right)\right)_{k=1}^{+\infty} \in S\left(\mathcal{P}_{0}, q_{0}, l_{0}\right)
$$

if and only if there exist sequences of matrix- and vector-functions, $\Phi_{k} \in$ $\widetilde{C}\left([a, b] ; \mathbb{R}^{n \times n}\right)(k=1,2, \ldots)$ and $\varphi_{k} \in \widetilde{C}\left([a, b] ; \mathbb{R}^{n}\right)(k=1,2, \ldots)$, respectively, such that

$$
\lim _{k \rightarrow+\infty} \sup \int_{a}^{b}\left\|\mathcal{P}_{k}^{*}(\tau)\right\| d \tau<+\infty
$$

and

$$
\begin{aligned}
& \lim _{k \rightarrow+\infty} \Phi_{k}(t)=0, \\
& \lim _{k \rightarrow+\infty} \varphi_{k}(t)=0, \\
& \lim _{k \rightarrow+\infty} \int_{a}^{t} \mathcal{P}_{k}^{*}(\tau) d \tau=\int_{a}^{t} \mathcal{P}_{0}(\tau) d \tau, \\
& \lim _{k \rightarrow+\infty} \int_{a}^{t} q_{k}^{*}(\tau) d \tau=\int_{a}^{t} q_{0}(\tau) d \tau
\end{aligned}
$$

uniformly on $[a, b]$, where

$$
\begin{aligned}
& \mathcal{P}_{k}^{*}(t) \equiv\left[E-\Phi_{k}(t)\right] \cdot \mathcal{P}_{k}(t)-\Phi_{k}^{\prime}(t), \\
& q_{k}^{*}(t) \equiv\left[E-\Phi_{k}(t)\right]\left[\mathcal{P}_{k}(t) \varphi_{k}(t)+q_{k}(t)-\varphi_{k}^{\prime}(t)\right] .
\end{aligned}
$$

Theorem 1'. Let (7)and (8) be satisfied. The (9) holds if and only if there exist sequences of matrix- and vector-functions, $\Phi_{k} \in \widetilde{C}\left([a, b] ; \mathbb{R}^{n \times n}\right)$ $(k=1,2, \ldots)$ and $\varphi_{k} \in \widetilde{C}\left([a, b] ; \mathbb{R}^{n}\right)(k=1,2, \ldots)$, respectively, such that

$$
\lim _{k \rightarrow+\infty} \sup \int_{a}^{b}\left\|\mathcal{P}_{k}^{*}(\tau)-\operatorname{diag} \mathcal{P}_{k}^{*}(\tau)\right\| d \tau<+\infty
$$


and the conditions (11)-(13) and

$$
\begin{gathered}
\lim _{k \rightarrow+\infty} \int_{a}^{t} \exp \left(-\int_{a}^{\tau} \operatorname{diag} \mathcal{P}_{k}^{*}(s) d s\right) \cdot q_{k}^{*}(\tau) d \tau= \\
=\int_{a}^{t} \exp \left(-\int_{a}^{\tau} \operatorname{diag} \mathcal{P}_{0}(s) d s\right) \cdot q_{0}(\tau) d \tau
\end{gathered}
$$

are fulfilled uniformly on $[a, b]$, where

$$
\mathcal{P}_{k}^{*}(t) \equiv\left[\mathcal{P}_{k}(t)-\Phi_{k}(t) \mathcal{P}_{k}(t)-\Phi_{k}^{\prime}(t)\right] \cdot\left[E-\Phi_{k}(t)\right]^{-1}
$$

and $q_{k}^{*}(t)$ is the vector-function defined by (16).

Before proving this theorems, we shall give a theorem from [1] and some of its generalizations.

Theorem $\mathbf{2}_{0}$. Let the conditions (6)-(8),

$$
\lim _{k \rightarrow+\infty} \sup \int_{a}^{b}\left\|\mathcal{P}_{k}(\tau)\right\| d \tau<+\infty
$$

hold and let the following conditions

$$
\begin{aligned}
& \lim _{k \rightarrow+\infty} \int_{a}^{t} \mathcal{P}_{k}(\tau) d \tau=\int_{a}^{t} \mathcal{P}_{0}(\tau) d \tau, \\
& \lim _{k \rightarrow+\infty} \int_{a}^{t} q_{k}(\tau) d \tau=\int_{a}^{t} q_{0}(\tau) d \tau
\end{aligned}
$$

hold uniformly on $[a, b]$. Then (9) is satisfied ${ }^{1}$.

Theorem 2. Let there exist sequences of matrix- and vector-functions, $\Phi_{k} \in \widetilde{C}\left([a, b] ; \mathbb{R}^{n \times n}\right)(k=1,2, \ldots)$ and $\varphi_{k} \in \widetilde{C}\left([a, b] ; \mathbb{R}^{n}\right)(k=1,2, \ldots)$, respectively, such that the conditions (10),

$$
\lim _{k \rightarrow+\infty}\left[c_{k}-l_{k}\left(\varphi_{k}\right)\right]=c_{0}
$$

hold and let the conditions (11),(13),(14) be fulfilled uniformly on $[a, b]$, where $\mathcal{P}_{k}^{*}(t)$ and $q_{k}^{*}(t)$ are the matrix- and vector-functions defined by (15) and (16), respectively. Let, moreover, conditions (7),(8) hold. Then for any sufficiently large $k$ the problem (3),(4) has the unique solution $x_{k}$ and

$$
\lim _{k \rightarrow+\infty}\left\|x_{k}-\varphi_{k}-x_{0}\right\|_{c}=0 .
$$

\footnotetext{
${ }^{1}$ See [1], Theorem 1.2.
} 
Proof. The transformation $z=x-\varphi_{k}$ reduces the problem (3),(4) to

$$
\begin{aligned}
& \frac{d z}{d t}=\mathcal{P}_{k}(t) z+r_{k}(t), \\
& l_{k}(z)=c_{k 1},
\end{aligned}
$$

where $r_{k}(t) \equiv \mathcal{P}_{k}(t) \varphi_{k}(t)+q_{k}(t)-\varphi_{k}^{\prime}(t), c_{k 1}=c_{k}-l_{k}\left(\varphi_{k}\right)(k=1,2, \ldots)$.

Let us show that for any sufficiently large $k$ the homogeneous problem $\left(3_{0}\right),\left(4_{0}\right)$ has only trivial solution.

Suppose this proposition is invalid. It can be assumed without loss of generality that for every natural $k$ the problem $\left(3_{0}\right),\left(4_{0}\right)$ has the solution $x_{k}$ for which

$$
\left\|x_{k}\right\|_{c}=1 .
$$

Moreover, it is evident that the vector-function $x_{k}$ is the solution of the system

$$
\frac{d x}{d t}=\mathcal{P}_{k}^{*}(t) x+\left[\Phi_{k}(t) \cdot x_{k}(t)\right]^{\prime} .
$$

According to (11) and (26)

$$
\lim _{k \rightarrow+\infty}\left[\Phi_{k}(t) \cdot x_{k}(t)\right]=0
$$

uniformly on $[a, b]$. Therefore the conditions of Theorem $2_{0}$ are fulfilled for the sequence of problems $(27),\left(4_{0}\right)$. Hence

$$
\lim _{k \rightarrow+\infty}\left\|x_{k}\right\|_{c}=0
$$

which contradicts $(26)$. This proves that the problem $\left(3_{0}\right),\left(4_{0}\right)$ has only trivial solution.

From this fact it follows that for any sufficiently large $k$ the problem $(24),(25)$ has the unique solution $z_{k}$.

It can easily be shown that the vector-function $z_{k}$ satisfies the system

$$
\frac{d z}{d t}=\mathcal{P}_{k}^{*}(t) z+r_{k}^{*}(t)
$$

where $r_{k}^{*}(t)=\left[\Phi_{k}(t) \cdot z_{k}(t)\right]^{\prime}+q_{k}^{*}(t)$.

Show that

$$
\lim _{k \rightarrow+\infty} \sup \left\|z_{k}\right\|_{c}<+\infty
$$

Let this proposal be invalid. Assume without loss of generality that

$$
\lim _{k \rightarrow+\infty}\left\|z_{k}\right\|_{c}=+\infty
$$


Put

$$
u_{k}(t)=\left\|z_{k}\right\|_{c}^{-1} \cdot z_{k}(t) \text { for } t \in[a, b] \quad(k=1,2, \ldots) .
$$

Then in view of (25) and (28), for every natural $k$ the vector-function $u_{k}(t)$ will be the solution of the boundary value problem

$$
\begin{aligned}
& \frac{d u}{d t}=\mathcal{P}_{k}^{*}(t) u+s_{k}(t), \\
& l_{k}(u)=\left\|z_{k}\right\|_{c}^{-1} \cdot c_{k 1},
\end{aligned}
$$

where $s_{k}(t)=\left\|z_{k}\right\|_{c}^{-1} \cdot r_{k}^{*}(t)$. Equations (11),(14),(23), and (30) imply

$$
\lim _{k \rightarrow+\infty}\left[\left\|z_{k}\right\|_{c}^{-1} \cdot c_{k 1}\right]=0
$$

and

$$
\lim _{k \rightarrow+\infty} \int_{a}^{t} s_{k}(\tau) d \tau=0
$$

uniformly on $[a, b]$. Hence, according to (10) and (13), the conditions of Theorem $2_{0}$ are fulfilled for the sequence of the last boundary value problems. Therefore

$$
\lim _{k \rightarrow+\infty}\left\|u_{k}\right\|_{c}=0 .
$$

This equality contradicts the conditions $\left\|u_{k}\right\|_{c}=1(k=1,2, \ldots)$. The inequality (29) is proved.

In view of (11),(14), and (29)

$$
\lim _{k \rightarrow+\infty} \int_{a}^{t} r_{k}^{*}(\tau) d \tau=\int_{a}^{t} q_{0}(\tau) d \tau
$$

uniformly on $[a, b]$.

Applying Theorem $2_{0}$ to the sequence of the problems (28),(25), we again show that

$$
\lim _{k \rightarrow+\infty}\left\|z_{k}-x_{0}\right\|_{c}=0 .
$$

Corollary 1. Let (6)-(8),

$$
\lim _{k \rightarrow+\infty} \sup _{\int_{a}^{b}}^{b} \mathcal{P}_{k}(\tau)-\Phi_{k}(\tau) \mathcal{P}_{k}(\tau)-\Phi_{k}^{\prime}(\tau) \| d \tau<+\infty
$$

hold and let the conditions (11),(21),(22),

$$
\lim _{k \rightarrow+\infty} \int_{a}^{t} \Phi_{k}(\tau) \mathcal{P}_{k}(\tau) d \tau=\int_{a}^{t} \mathcal{P}^{*}(\tau) d \tau
$$

and

$$
\lim _{k \rightarrow+\infty} \int_{a}^{t} \Phi_{k}(\tau) q_{k}(\tau) d \tau=\int_{a}^{t} q^{*}(\tau) d \tau
$$


be fulfilled uniformly on $[a, b]$, where $\Phi_{k} \in \widetilde{C}\left([a, b] ; \mathbb{R}^{n \times n}\right)(k=1,2, \ldots)$, $\mathcal{P}^{*} \in L\left([a, b] ; \mathbb{R}^{n \times n}\right), q^{*} \in L\left([a, b] ; \mathbb{R}^{n}\right)$. Let, moreover, the system

$$
\frac{d x}{d t}=\mathcal{P}_{0}^{*}(t) x+q_{0}^{*}(t),
$$

where $\mathcal{P}_{0}^{*}(t) \equiv \mathcal{P}_{0}(t)-\mathcal{P}^{*}(t), q_{0}^{*}(t) \equiv q_{0}(t)-q^{*}(t)$, have a unique solution satisfying the condition (2). Then

$$
\left(\left(\mathcal{P}_{k}, q_{k}, l_{k}\right)\right)_{k=1}^{+\infty} \in S\left(\mathcal{P}_{0}^{*}, q_{0}^{*}, l_{0}\right) .
$$

Proof. It suffices to assume in Theorem 2 that $\varphi_{k}(t) \equiv 0$ and to notice that

$$
\lim _{k \rightarrow+\infty} \int_{a}^{t}\left[E-\Phi_{k}(\tau)\right] \cdot \mathcal{P}_{k}(\tau) d \tau=\int_{a}^{t} \mathcal{P}_{0}^{*}(\tau) d \tau
$$

and

$$
\lim _{k \rightarrow+\infty} \int_{a}^{t}\left[E-\Phi_{k}(\tau)\right] \cdot q_{k}(\tau) d \tau=\int_{a}^{t} q_{0}^{*}(\tau) d \tau
$$

uniformly on $[a, b]$.

Corollary 2. Let (6)-(8) hold, and let there exist a natural number $m$ and matrix-functions $\mathcal{P}_{o j} \in L\left([a, b], \mathbb{R}^{n \times n}\right)(j=1, \ldots, m)$ such that

$$
\begin{aligned}
& \lim _{k \rightarrow+\infty}\left[\mathcal{P}_{k m}(t)-\mathcal{P}_{k}(t)\right]=0, \\
& \lim _{k \rightarrow+\infty} \int_{a}^{t}\left[E+\mathcal{P}_{k m}(\tau)-\mathcal{P}_{k}(\tau)\right] \cdot \mathcal{P}_{k}(\tau) d \tau=\int_{a}^{t} \mathcal{P}_{0}(\tau) d \tau, \\
& \lim _{k \rightarrow+\infty} \int_{a}^{t}\left[E+\mathcal{P}_{k m}(\tau)-\mathcal{P}_{k}(\tau)\right] \cdot q_{k}(\tau) d \tau=\int_{a}^{t} q_{0}(\tau) d \tau
\end{aligned}
$$

uniformly on $[a, b]$, where

$$
\begin{gathered}
\mathcal{P}_{k 1}(t) \equiv \mathcal{P}_{k}(t), \quad \mathcal{P}_{k j+1}(t) \equiv \mathcal{P}_{k j}(t)-\int_{a}^{t}\left[\mathcal{P}_{k j}(\tau)-\mathcal{P}_{0 j}(\tau)\right] d \tau \\
(j=1, \ldots, m) .
\end{gathered}
$$

Let, moreover,

$$
\begin{aligned}
\lim _{k \rightarrow+\infty} \sup \int_{a}^{b} \|\left[E+\mathcal{P}_{k m}(\tau)-\mathcal{P}_{k}(\tau)\right] \cdot \mathcal{P}_{k}(\tau)+ \\
+\left[\mathcal{P}_{k m}(\tau)-\mathcal{P}_{k}(t)\right]^{\prime} \| d \tau<+\infty
\end{aligned}
$$

Then (9) holds. 
Theorem $2^{\prime}$. Let there exist sequences of matrix- and vector-functions, $\Phi_{k} \in \widetilde{C}\left([a, b] ; \mathbb{R}^{n \times n}\right)(k=1,2, \ldots)$ and $\varphi_{k} \in \widetilde{C}\left([a, b] ; \mathbb{R}^{n}\right)(k=1,2, \ldots)$, respectively, such that the conditions (17),(23) hold, and let the conditions $(11),(13)$, and (18) be fulfilled uniformly on $[a, b]$. Here $\mathcal{P}_{k}^{*}(t)$ and $q_{k}^{*}(t)$ are the matrix- and vector-functions defined by (19) and (16), respectively. Then the conclusion of Theorem 2 is true.

Proof. In view of (14), we may assume without loss of generality that for every natural $k$ the matrix $E-\Phi_{k}(t)$ is invertible for $t \in[a, b]$.

For every $k \in\{0,1, \ldots\}$ and $t \in[a, b]$ assume

$$
\begin{gathered}
\mathcal{P}_{0}^{*}(t)=\mathcal{P}_{0}(t), \quad q_{0}^{*}(t)=q_{0}(t), \quad \Phi_{0}(t)=0, \quad \varphi_{0}(t)=0, \\
c_{k 1}=c_{k}-l_{k}\left(\varphi_{k}\right), \quad Q_{k}(t)=H_{k}(t) \cdot\left[\mathcal{P}_{k}^{*}(t)-\operatorname{diag} \mathcal{P}_{k}^{*}(t)\right] \cdot H_{k}^{-1}(t), \\
r_{k}(t)=H_{k}(t) \cdot q_{k}^{*}(t),
\end{gathered}
$$

where

$$
H_{k}(t)=\exp \left(-\int_{a}^{t} \operatorname{diag} \mathcal{P}_{k}^{*}(\tau) d \tau\right)
$$

Moreover, assume

$$
l_{k}^{*}(z)=l_{k}(x) \text { for } z \in C\left([a, b] ; \mathbb{R}^{n}\right),
$$

where $x(t)=\left[E-\Phi_{k}(t)\right]^{-1} \cdot H_{k}^{-1}(t) \cdot z(t)$.

From (13) it follows that $l_{k}^{*}: C\left([a, b] ; \mathbb{R}^{n}\right) \rightarrow \mathbb{R}^{n}(k=0,1, \ldots)$ is a sequence of linear continuous operators for which conditions (7) and (8) are satisfied.

For every $k \in\{0,1, \ldots\}$ the transformation

$$
z(t)=H_{k}(t) \cdot\left[E-\Phi_{k}(t)\right] \cdot\left[x(t)-\varphi_{k}(t)\right] \text { for } t \in[a, b]
$$

reduces the problem (3),(4) to

$$
\begin{aligned}
& \frac{d z}{d t}=Q_{k}(t) z+r_{k}(t), \\
& l_{k}^{*}(z)=c_{k 1}
\end{aligned}
$$

and the problem (1),(2) to

$$
\begin{aligned}
& \frac{d z}{d t}=Q_{0}(t) z+r_{0}(t), \\
& l_{0}^{*}(z)=c_{0} .
\end{aligned}
$$

In view of (13) and (17) from Lemma $1.1([1]$, p.9) it follows that

$$
\lim _{k \rightarrow+\infty} \int_{a}^{t} Q_{k}(\tau) d \tau=\int_{a}^{t} Q_{0}(\tau) d \tau
$$


uniformly on $[a, b]$. According to Theorem $2_{0}$ from the above and from $(7),(8),(17),(18),(23)$ it follows that the problem $(32),(33)$ has the unique solution $z_{k}$ for any sufficiently large $k$, and

$$
\lim _{k \rightarrow+\infty}\left\|z_{k}-z_{0}\right\|_{c}=0
$$

where $z_{0}$ is the unique solution of the problem (34),(35). Therefore (11),(13) and (31) show that the statement of the theorem is true.

Corollary 3. Let the conditions (6)-(8),

$$
\lim _{k \rightarrow+\infty} \sup \int_{a}^{b}\left\|\mathcal{P}_{k}(\tau)-\operatorname{diag} \mathcal{P}_{k}(\tau)\right\| d \tau<+\infty
$$

hold and let (21) and

$$
\begin{gathered}
\lim _{k \rightarrow+\infty} \int_{a}^{t} \exp \left(-\int_{a}^{\tau} \operatorname{diag} \mathcal{P}_{k}(s) d s\right) \cdot q_{k}(\tau) d \tau= \\
=\int_{a}^{t} \exp \left(-\int_{a}^{\tau} \operatorname{diag} \mathcal{P}_{0}(s) d s\right) \cdot q_{0}(\tau) d \tau
\end{gathered}
$$

be fulfilled uniformly on $[a, b]$. Then (9) holds.

Remark. As compared with Theorem $2_{0}$ and the results of [2], it is not assumed in Theorems 2 and $2^{\prime}$ that the equalities (21) and (22) hold uniformly on $[a, b]$. Below we will give an example of a sequence of boundary value problems for linear systems for which (9) holds but (21) is not fulfilled uniformly on $[a, b]$.

Example. Let $a=0, b=2 \pi, n=2$, and for every natural $k$ and $t \in[0,2 \pi]$, let

$$
\begin{aligned}
& \mathcal{P}_{k}(t)=\left(\begin{array}{cc}
0 & p_{k 1}(t) \\
0 & p_{k 2}(t)
\end{array}\right), \quad \mathcal{P}_{0}(t)=\left(\begin{array}{ll}
0 & 0 \\
0 & 0
\end{array}\right), \\
& \varphi_{k}(t)=q_{k}(t)=q_{0}(t)=\left(\begin{array}{l}
0 \\
0
\end{array}\right) \text {; } \\
& p_{k 1}(t)= \begin{cases}(\sqrt{k}+\sqrt[4]{k}) \sin k t & \text { for } t \in I_{k}, \\
\sqrt{k} \sin k t & \text { for } t \in[0,2 \pi] \backslash I_{k} ;\end{cases} \\
& p_{k 2}(t)= \begin{cases}-\alpha_{k}^{\prime}(t) \cdot\left[1-\alpha_{k}(t)\right]^{-1} & \text { for } t \in I_{k}, \\
0 & \text { for } t \in[0,2 \pi] \backslash I_{k}\end{cases} \\
& \beta_{k}(t)=\int_{0}^{t}\left[1-\alpha_{k}(\tau)\right] \cdot p_{k 1}(\tau) d \tau \\
& \alpha_{k}(t)= \begin{cases}4 \pi^{-1}(\sqrt[4]{k}+1)^{-1} \sin k t & \text { for } t \in I_{k}, \\
0 & \text { for } t \in[0,2 \pi] \backslash I_{k},\end{cases}
\end{aligned}
$$


where $\left.I_{k}=\cup_{m=0}^{k-1}\right] 2 m k^{-1} \pi,(2 m+1) k^{-1} \pi[$. Let, moreover, for every $k \in$ $\{0,1, \ldots\}, Y_{k}(t)$ be the fundamental matrix of the system $\left(3_{0}\right)$ satisfying

$$
Y_{k}(a)=E .
$$

It can easily be shown that for every natural $k$ we have

$$
Y_{0}(t)=E, \quad Y_{k}(t)=\left(\begin{array}{cc}
1 & \beta_{k}(t) \\
0 & 1-\alpha_{k}(t)
\end{array}\right) \text { for } t \in[0,2 \pi]
$$

and

$$
\lim _{k \rightarrow+\infty} Y_{k}(t)=Y_{0}(t)
$$

uniformly on $[0,2 \pi]$, since

$$
\lim _{k \rightarrow+\infty}\left\|\alpha_{k}\right\|_{c}=\lim _{k \rightarrow+\infty}\left\|\beta_{k}\right\|_{c}=0 .
$$

Note that

$$
\lim _{k \rightarrow+\infty} \int_{0}^{2 \pi} p_{k 1}(t) d t=2 \lim _{k \rightarrow+\infty} \sqrt[4]{k}=+\infty .
$$

Therefore neither the conditions of Theorem $2_{0}$ nor the results of [2] are fulfilled.

On the other hand, if we assume that

$$
\Phi_{k}(t)=E-Y_{k}^{-1}(t) \text { for } t \in[0,2 \pi](k=1,2, \ldots),
$$

then the conditions of Theorems 2 and $2^{\prime}$ will be fulfilled, and if we put

$$
\Phi_{k}(t)=\left(\begin{array}{cc}
\alpha_{k}(t) & \beta_{k}(t) \\
0 & 0
\end{array}\right) \text { for } t \in[0,2 \pi](k=1,2, \ldots),
$$

then in this case only the conditions of Theorem 2 will be fulfilled, since

$$
\lim _{k \rightarrow+\infty} \sup \int_{0}^{2 \pi}\left|p_{k 2}(t)\right| d t=+\infty
$$

Proof of Theorem 1. The sufficiency follows from Theorem 2, since in view of (6),(8), and (12), condition (23) holds.

Let us show the necessity. Let $c_{k} \in \mathbb{R}^{n}(k=0,1, \ldots)$ be an arbitrary sequence satisfying (6) and let $e_{j}=\left(\delta_{i j}\right)_{i=1}^{n}(j=1, \ldots, n)$, where $\delta_{i j}=1$ if $i=j$ and $\delta_{i j}=0$ if $i \neq j$.

In view of (9), we may assume without loss of generality that for every natural $k$ the problem (3),(4) has the unique solution $x_{k}$.

For any $k \in\{0,1, \ldots\}$ and $j \in\{1, \ldots, m\}$ assume

$$
y_{k j}(t)=x_{k}(t)-x_{k j}(t) \quad(t \in[a, b])
$$


where $x_{o j}$ and $x_{k j}(k=1,2, \ldots)$ are the unique solutions of (1) and (3) satisfying

$$
l_{0}(x)=c_{0}-e_{j} \text { and } l_{k}(x)=c_{k}-e_{j},
$$

respectively. Moreover, for every $k \in\{0,1, \ldots\}$ denote by $Y_{k}(t)$ the matrixfunction whose columns are $y_{k 1}(t), \ldots, y_{k n}(t)$.

It can easily be shown that $y_{o j}$ and $y_{k j}$ satisfy $\left(1_{0}\right)$ and $\left(3_{0}\right)$, respectively, and

$$
l_{k}\left(y_{k j}\right)=e_{j} \quad(j=1, \ldots, n ; k=0,1, \ldots) .
$$

If for some $k$ and $\alpha_{j} \in \mathbb{R}(j=1, \ldots, n)$

$$
\sum_{j=1}^{n} \alpha_{j} y_{k j}(t)=0 \quad(t \in[a, b])
$$

then, using (36), we have

$$
\sum_{j=1}^{n} \alpha_{j} e_{j}=0
$$

and therefore

$$
\alpha_{1}=\cdots=\alpha_{n}=0,
$$

i.e., $Y_{0}$ and $Y_{k}$ are the fundamental matrices of the systems $\left(1_{0}\right)$ and $\left(3_{0}\right)$, respectively. Hence, (5) implies

$$
\lim _{k \rightarrow+\infty} Y_{k}^{-1}(t)=Y_{0}^{-1}(t) \text { uniformly on }[a, b] .
$$

Let, for every natural $k$ and $t \in[a, b]$,

$$
\begin{gathered}
\Phi_{k}(t)=E-Y_{0}(t) Y_{k}^{-1}(t), \\
\varphi_{k}(t)=x_{k}(t)-x_{0}(t) .
\end{gathered}
$$

Let us show (10)-(14). Equations (11) and (12) are evident. Moreover, using the equality

$$
\left[Y_{k}^{-1}(t)\right]^{\prime}=-Y_{k}^{-1}(t) \mathcal{P}_{k}(t) \text { for } t \in[a, b](k=1,2, \ldots),
$$

it can be easily shown that

$$
\mathcal{P}_{k}^{*}(t)=\mathcal{P}_{0}(t) Y_{0}(t) Y_{k}^{-1}(t) \quad \text { for } t \in[a, b](k=1,2, \ldots)
$$

and

$$
\begin{gathered}
\int_{a}^{t} q_{k}^{*}(\tau) d \tau=Y_{0}(t) Y_{k}^{-1}(t) x_{0}(t)-Y_{0}(a) Y_{k}^{-1}(a) x_{0}(a)- \\
-\int_{a}^{t} \mathcal{P}_{0}(\tau) Y_{0}(\tau) Y_{k}^{-1}(\tau) x_{0}(\tau) d \tau \text { for } t \in[a, b](k=1,2, \ldots) .
\end{gathered}
$$


Therefore, according to (37) the conditions (10),(13), and (14) are fulfilled uniformly on $[a, b]$. This completes the proof.

The proof of Theorem $1^{\prime}$ is analogous. We note only that $\Phi_{k}$ and $\varphi_{k}$ are defined as above.

The behavior at $k \rightarrow+\infty$ of the solution of the Cauchy problem $\left(l_{k}(x)=\right.$ $\left.x\left(t_{0}\right), t_{0} \in[a, b]\right)$ and of the Cauchy-Nicoletti problem $\left(l_{k}(x)=\left(x_{i}\left(t_{i}\right)\right)_{i=1}^{n}\right.$, $\left.t_{i} \in[a, b]\right)$ is considered in [3-5]. Moreover, in [6] the necessary conditions for the stability of the Cauchy problem are investigated.

\section{REFERENCES}

1. I.T.Kiguradze, Boundary value problems for systems of ordinary differential equations. (Russian) Modern problems in mathematics. The latest achievements (Itogi nauki i tekhniki. VINITI Acad. Sci. USSR). Moscow, 1987, V.30, 3-103.

2. M.T.Ashordia and D.G.Bitsadze, On the correctness of linear boundary value problems for systems of ordinary differential equations. (Russian) Bull. Acad. Sci. Georgian SSR 142(1991), No. 3, 473-476.

3. D.G.Bitsadze, On the problem of dependence on the parameter of the solution of multipoint nonlinear boundary value problems. (Russian) Proc. I.N.Vekua Inst. Appl. Math. Tbilis. State Univ. 22(1987), 42-55.

4. J.Kurzweil and J.Jarnik, Iterated Lie brackets in limit processes in ordinary differential equations. Results in Mathematics, Birkhäuser Verlag. Basel, 1988, V.14, 125-137.

5. A.M.Samoilenko, Investigation of differential equations with "non-regular" right part. (Russian) Abhandl. der Deutsch. Akad. Wiss. Berlin. Kl. Math., Phys. und Tech. 1965, No. 1, 106-113.

6. N.N.Petrov, Necessary conditions of continuity with respect to the parameter for some classes of equations. (Russian) Vestnik Leningrad. Univ. Mat. Mech. Astronom. (1965), No. 1, 47-53.

(Received 22.09.1992)

Author's address:

I.Vekua Institute of Applied Mathematics of Tbilisi State University

2 University St., 380043 Tbilisi

Republic of Georgia 